\title{
Gynécomastie Révélant Une Hyperthyrö̈die (A Propos D'un Cas) Gynecomastia Reaveling Hyperthyroidism (About One Case)
}

\author{
Houda Salhi ${ }^{1}$, Imane Yassine, Hanane El Ouahabi ${ }^{1}$, Farida Ajdi $^{2}$ \\ ${ }^{I}$ Service d'Endocrinologie-Diabétologie. CHU Hassan II, Faculté De Médecine Et De Pharmacie, Université \\ Sidi Mohamed Ben Abdellah, Fès, Maroc. \\ ${ }^{2}$ Professeur De l'Enseignement Supérieur Endocrinologie Et Maladies Métaboliques. Faculté De Médecine Et \\ De Pharmacie Université Ibn Zohr, Agadir. Maroc
}

\begin{abstract}
Although gynaecomastia is a well-documented manifestation among male patients with hyperthyroidism, it is extremely rare to present as the initial or chief complaint in a subject with undiagnosed hyperthyroidism. However the association of neuropathy with thyrotoxicosis is not frequently recognized. We report a case of a 28-year-old, who was followed for idiopathic poly neuropathy complicated by paraplegia. Examination revealed bilateral gynaecomastia without symptom of hyperthyroidism. Hyperthyroidism was confirmed biochemically secondary to nodular goiter. Treatment of thyrotoxicosis resulted in resolution of the gynaecomastia and probably his neuropathy.

Résumé: Bien que la gynécomastie soit une manifestation bien documentée chez les patients masculins souffrant d'hyperthyrö̈die, il est extrêmement rare qu'il soit le seul signe révélateur chez un patient souffrant d'une hyperthyroïdie non diagnostiquée. L'hyperthyrö̈die peut être une cause d'une poly neuropathie malgré que ce soit peu connu. Nous rapportons le cas d'un jeune homme de 28 ans suivie pour une poly neuropathie étiquetée comme idiopathique responsable de paraplégie, admis pour bilan d'une gynécomastie bilatérale d'installation récente, chez qui l'examen ne révèle pas de signes d'hyperthyrö̈die, gynécomastie stade 3. Par ailleurs, le bilan révèle une hyperthyroïdie biologique sur probable goitre nodulaire. Le traitement par antithyrö̈dien de synthèse pourra améliorer la gynécomastie et probablement sa neuropathie au cours ou dans l'année qui suit le traitement.
\end{abstract}

Mots clés: hyperthyroidie, gynécomastie, polyneuropathie

Keyswords: hyperthyroidism, gynécomatia, polyneuropathy

\section{Introduction}

Les manifestations clinique de l'hyperthyroïdie sont constantes mais d'intensité variable d'un malade à l'autre. Elle peut se manifester par des signes usuels tels les palpitations, la perte de poids, des tremblements, des sueurs, intolérance à la chaleur, la diarrhée, exophtalmie. Une association gynécomastie et hyperthyroïdie peut être présente dans $40 \%$ des cas chez l'homme [1,2]. Bien que l'association et hyperthyroïdie est bien connu, cependant sa présentant autant qu'un symptôme révélateur de l'hyperthyroïdie reste extrêmement rare [3].

\section{Observation}

Il s'agit d'un patient âgé de 28 ans, suivi depuis 5 ans pour une poly neuropathie d'étiologie inconnue (dont le bilan biologique et morphologique n'a pas objectivé une cause évidente), sans notion de prise médicamenteuse, ayant consulté pour une gynécomastie bilatérale d'installation récente. L'examen clinique trouve un patient paraplégique, en euthyroidie clinique, présence d'une gynécomastie bilatérale stade 3 selon la classification de Simon, absence de signes d'hypoandrisme associés, ou de signes orientant vers une insuffisance hépatique. Le bilan biologique avait montré une fonction rénale et une fonction hépatique correcte, une testostéronémie normale à $6,82 \mathrm{ng} / \mathrm{ml}$, avec une $\mathrm{FSH}$, LH correctes et un œstradiol correct. Une prolactinémie normale à $9.68 \mathrm{ng} / \mathrm{ml}$. Les marqueurs tumoraux (BHCG et AFP) étaient négatifs et l'échographie testiculaire était sans particularité. Par ailleurs, le bilan thyroïdien a révélé une hyperthyroïdie périphérique avec une TSH freinée à $0,010 u u i / m l$ avec une LT4 élevée $(1,68 \mathrm{ng} / \mathrm{l}$ pour des normes allant de 0,7 a 1,48), le bilan immunologique (dosage des anticorps anti récepteurs et anti thyroperoxydase) était normal. L'échographie cervicale a été visualisé un goitre avec de multiples nodules dont le plus grand mesurant $4 \mathrm{~mm}$, le patient fut mis sous antithyroïdiens de synthèse. Le patient fut readressé en neurologie pour prise en charge de sa polyneuropathie qui pourra être une manifestation de l'hyperthyroïdie. 


\section{Discussion}

La cause de la gynécomastie, peut être physiologique (nouveau né, adolescent et sujet âgé) et non physiologique (hypogonadisme, prise médicamenteuse, insuffisance hépatique ou rénale chronique, hyperthyroïdie....). Bien que 10-40\% des patients souffrant d'hyperthyroïdie peuvent présenter une gynécomastie mais sa présence autant que le seul symptôme révélateur de l'hyperthyroïdie reste rarement décrit [4,5]. Notre patiente était asymptomatique sur le plan clinique alors qu'il avait une gynécomastie classée stade 3 selon la classification de Simon et le bilan biologique qui a objectivé une TSHus freinée avec une élévation de la LT4 confirmant le diagnostic de l'hyperthyroïdie. Chez l'homme, la gynécomastie résulte d'un déséquilibre de la balance ostrogènes circulants (augmentation) et /ou de la testostérone circulante (diminution) ce qui induit une stimulation du tissu mammaire [6]. La progestérone peut avoir un effet additif en améliorant la réponse de la glande mammaire aux effets des œstrogènes [7]. Dans l'hyperthyrö̈die, un changement du profil des hormones sexuelles chez l'homme est bien constaté avec une élévation de la testostérone totale, SHBG, dihyrotestosterone, œstradiol total ainsi que la LH [8,9]. L'augmentation de la SHBG conduit à une réduction du taux de testostérone libre. Le dosage des hormones sexuelles à savoir la testostérone totale, l'œstradiol, FSH LH et prolactine sont revenus normaux, par contre le taux de la testosterone libre ainsi que SHBG n'a pas été effectué. Par ailleurs, il a été remarqué aussi que le taux d'androstenediole et le sulfate androstenediole qui sont des métabolites actifs de déhydroépiandrostérone (DHEA) et du sulfate de DHEA, respectivement ont une activité œstrogènique [10]. Le taux de ses stéroïdes a été retrouvé augmenté en cas d'hyperthyroïdie [7]. Dans la plupart des cas, la gynécomastie régresse au cours ou dans l'année qui suit le traitement de l'hyperthyroïdie. Par ailleurs, plusieurs maladies neurologiques comme la myopathie, paralysie périodique, ophtalmoplégie et la myasthénie sont connu par leur association avec l'hyperthyroïdie. Par contre l'association entre neuropathie et hyperthyroïdie est peu connu [11] et la première description de cette association fut faite par Charcot en 1889[12]. Le traitement par antithyroïdiens de synthèse améliore l'hyperthyroïdie mais aussi la neuropathie [13].notre patient est suivie pour une poly neuropathie idiopathique compliquée de paraplégie depuis 05ans et la découverte de l'hyperthyroïdie pourrai expliquer la présence de la gynécomastie mais probablement la présence de la poly neuropathie

\section{Conclusion}

Notre cas démontre que la gynécomastie malgré sa rareté peut être un signe révélateur d'une hyperthyroïdie asymptomatique sur le plan clinique. En outre, même si peu décrit, la neuropathie peut être une conséquence d'une hyperthyroïdie dont il faut y penser. Le traitement de l'hyperthyroïdie par antithyroïdien de synthèse est le traitement clé de ses deux pathologies.

\section{Référence}

[1]. Becker KL, Winnacker JL,MatthewsMJ, Higgins GA. Gynecomastia and hyperthyroidism. An endocrine and histological investigation. J Clin Endocrinol Metab 1968; 28:277-85.

[2]. Ashkar FS, Smoak WM III, Gilson AJ, Miller R. Gynecomastia and mastoplasia in Graves' disease. Metabolism 1970; $19: 946-51$.

[3]. Ho HK, Loh KC. Hyperthyrodism with gynaecomastia as the initial complaint: a case report. Singapore, Ann. Acad. Med., 1998; 27:594-96.

[4]. Muthusamy E. Hyperthyroidism with gynaecomastia, galactorrhoea and periodic paralysis. Singapore Med J. 1991; 32(5):371-372.

[5]. Dickson G. Gynecomastia. Am Fam Physician. 2012 Apr1; 85(7):716-22.

[6]. ChanWB1, Yeung VT, Chow CC, SoWY,CockramCS.Gynaecomastia as a presenting feature of thyrotoxicosis. Postgrad Med J. 1999 Apr; 75(882):229-31.

[7]. Tauheed S1, Haque MN. Unilateral gynaecomastia--an unusual presentation of thyrotoxicosis. J Pak Med Assoc. 2002 May;52(5):227-8.

[8]. Chopra IJ, Tulchinsky D. Status of estrogen-androgen balance in hyperthyroid men with Graves' disease. J Clin Endocrinol Metab 1974; 38:269-77.

[9]. Chopra IJ. Gonadal steroids and gonadotrophins in hyperthyroidism. Med Clin North Am 1975; 59:1109-21.

[10]. Tagawa N1, Takano T, Fukata S, Kuma K, Tada H, Izumi Y, Kobayashi Y, Amino N. Serum concentration of androstenediol and androstenediol sulfate in patients with hyperthyroidism and hypothyroidism. Endocr J. 2001 Jun; 48(3):345-54.

[11]. Duyff RF, Van den Bosch J, Laman DM, van Loon BJ, Linssen WH. Neuromuscular findings in thyroid dysfunction: a prospective clinical and electrodiagnostic study. J Neurol Neurosurg Psychiatry. 2000; 68: 750-5.

[12]. Charcot J. Nouveaux signes de la maladie de Basedow. Bull Méd. 1889; 3: 147-49.

[13]. Sahni V1, Gupta N, Anuradha S, Tatke M, Kar P. Thyrotoxic neuropathy- an under diagnosed condition. Med J Malaysia. 2007 Mar; 62(1):76-7. 